\title{
Progression of diabetic retinopathy correlated with muscle perfusion disturbances of the lower limbs, with clinically important diagnostic recommendations
}

Wiesław Tryniszewski ${ }^{1}$, Mariusz Gadzicki ${ }^{1}$, Zbigniew Maziarz ${ }^{1}$, Jarosław Kuśmierczyk², Roman Goś², Jacek Rysz $z^{3}$ Maciej Banach ${ }^{3}$

1Department of Radiological, Isotopic Diagnostics and Therapy, Medical University of Lodz, Poland

${ }^{2}$ Department of Ophthalmology and Sight Rehabilitation, Medical University of Lodz, Poland

3Department of Nephrology, Hypertension and Family Medicine, Medical University of Lodz, Poland

Submitted: 14 July 2010

Accepted: 16 September 2010

Arch Med Sci 2010; 6, 6: 904-911

DOI: 10.5114/aoms.2010.19300

Copyright @ 2010 Termedia \& Banach

\section{Abstract}

Introduction: The objective of this study was to investigate the relationship between microvascular and macrovascular disturbances in patients with type 2 diabetes, as shown by results of ophthalmological examination and by vascular and perfusion examinations of the lower limbs.

Material and methods: A total of 85 patients with type 2 diabetes and an additional cardiovascular risk factor were enrolled in the study. All patients underwent complex ophthalmological examination, including fundus colour photography and fluorescein angiography, and were divided into two groups: group I with signs of diabetic non-proliferative retinopathy (NPDR), and group II with signs of diabetic proliferative retinopathy (P/PDR). After collection of the general medical history and analysis of medical data, patients underwent vascular and muscle perfusion examination of the lower limbs.

Results: In the P/PDR group, disturbances of lower-limb perfusion were more frequent than in the NPDR group. Analysis of the blood flow and results of lowerlimb muscle perfusion for the two groups showed a significant relationship with the severity of microvascular complications observed in examination of the fundus.

Conclusions: Ophthalmological assessment of the progression of diabetic retinopathy in patients with type 2 diabetes is a reliable indicator of the changes in peripheral vessel systems and perfusion defects in the lower limbs.

Key words: Diabetic retinopathy, muscle perfusion disturbances, diabetes type 2

\section{Introduction}

Diabetes and its complications are important therapeutic problems. Early recognition and treatment of the vascular complications of type 2 diabetes, such as diabetic angiopathy, diabetic retinopathy, and muscle perfusion disturbances, are critical. The non-invasive and sensitive tests for detecting these complications include indirect ophthalmoscopy and fluorescein angiography of the retina, together with muscle perfusion scintigraphy of the lower limbs [1].

\author{
Corresponding author: \\ Wiesław Tryniszewski, MD \\ Department of Radiological, \\ Isotopic Diagnostics and \\ Therapy \\ Medical University of Lodz \\ Żeromskiego 113 \\ 90-549 Lodz, Poland \\ Phone/fax: +48426393690 \\ E-mail: \\ wieslaw.tryniszewski@umed. \\ lodz.pl
}


The definition of the relation between the progression of changes in the eyes and intensity of muscle perfusion in the legs is very important for a clinical diagnosis. Identification of such a relation would allow for a quick, non-invasive evaluation of the risk of both microangiopathic and macroangiopathic vascular complications in patients with type 2 diabetes [1, 2].

Therefore the aim of the study was to (1) define the correlation between the intensity of diabetic retinopathy and muscle perfusion disturbances in the legs of patients with type 2 diabetes; (2) assess the extent to which the progression of diabetic retinopathy can be a determinant of a perfusion impairment in patients with type 2 diabetes and thereby recommend appropriate diagnostic tests for lower-limb perfusion disturbances.

\section{Material and methods}

Eighty-five patients with type 2 diabetes aged 50 to 75 (41 women and 44 men) were included in the analysis.

Patients with glaucoma, senile macular degeneration, high myopia, and retinal diseases and those who had had interventions that precluded use of the typical classification of diabetic retinopathy were excluded from the study, as well as patients who had had myocardial infarction and stroke and surgical procedures involving the lowerlimb muscles. The duration of type 2 diabetes was 10 to 26 years $(16.88 \pm 4.54)$. The patients were selected on the basis of progression of retinopathy as defined by an ophthalmologist with more than 5 years' experience diagnosing retinopathy. On the basis of the progression of retinopathy, the patients were included in one of two groups. In each group, there were subgroups of patients treated with insulin and oral drugs:

Group 1 - NPDR (non-proliferative diabetic retinopathy) consisted of 43 patients (27 men and 16 women, aged 50-67) with changes typical of non-proliferative diabetic retinopathy. The inclusion criterion was the presence of at least one microaneurysm confirmed by ophthalmological examination, eye fundus photographs, or eye fundus fluorescein angiography. The group was divided into 2 subgroups depending on the type of diabetes treatment; the subgroup taking drugs orally consisted of 25 patients aged $57 \pm 4.51$ years, and the subgroup receiving insulin consisted of 18 patients aged $59 \pm 5.71$ years.

Group 2 - P/PDR (pre-proliferative and proliferative diabetic retinopathy) consisted of 42 patients (23 men and 19 women, aged 50-75) who had any eye fundus neovascularization characteristic for proliferative diabetic retinopathy in any location (neovascularization of the optic disc [NVD], neovascularization elsewhere [NVE]). The subgroup taking drugs orally consisted of 18 patients aged $63 \pm 7.61$ years, and the subgroup receiving insulin included 24 patients aged $65 \pm 7.61$ years.

All patients underwent subjective and objective physician assessment, as well as complex ophthalmological assessment. These included assessments of:

- general history, including age, sex, diabetes duration, past medical treatment;

- history concerning risk factors leading to circulation disturbances of lower limbs;

- history concerning eye diseases;

- ophthalmological examination, including best corrected visual acuity, intraocular pressure, full examination of the anterior segment of the eye, fundus examination by indirect ophthalmoscopy, digital colour fundus photography and fluorescein angiography.

The scale elaborated by the Global Diabetic Retinopathy Group was used for assessment of diabetic retinopathy progression [1].

Colour photography and fluorescein angiography were performed with a TRC 50EX Topcon fundus camera, Topcon, equipped with the lens system TL 211 and digital camera with resolution of 12 megapixels (Nikon D2).

Seven standard non-stereoscopic fundus pictures in the $30^{\circ}$ range according to the ETDRS standard were obtained. The presence of the following was assessed for entry to the study groups: microaneurysms, haemorrhages, hard exudates, venous beading, change in the width of venous vessels, intra-retinal microvascular abnormalities (IRMA), cotton wool spots, retinal oedema, neovascularization of optic disc (NVD) and retinal neovascularization elsewhere (NVE), according to standard ETDRS pictures.

In all patients the radioisotopic assessment of lower-limb muscle perfusion at rest was performed. Scintigraphic examination of lower-limb perfusion was performed with a gamma-camera after the intravenous administration of technetium 99m methoxy-isobutylisonitrate (MIBI Tc ${ }^{99 m}$ ), activity $11.1 \mathrm{MBq} / \mathrm{kg}$ b.m. (body mass); 5 min after the injection, a scintigram of the entire body was obtained, together with static scintigrams of lowerlimb muscles. The pictures were mathematically analysed with the Assessment Limb Lower Perfusion (ALLP) program, which defined the muscle perfusion indexes of stress and rest: WPLP indicated the perfusion index of the left tibial muscles; WPPP - perfusion index of the right tibial muscles; WPLU - perfusion index of the left femoral muscles; and WPPU - perfusion index of the right femoral muscles. The perfusion index values allowed for definition of the calf perfusion index (WPP) and thigh perfusion index (WPU) [2-4]. 
Table I. Range of normal values of the indexes in patients more than 50 years of age

\begin{tabular}{|lllll|}
\hline \multirow{2}{*}{$\begin{array}{l}\text { Normal } \\
\text { values range }\end{array}$} & \multicolumn{4}{c|}{ Muscle perfusion indexes $^{*}$} \\
\cline { 2 - 5 } & \multicolumn{2}{c}{ At rest } & \multicolumn{2}{c|}{ On exercise } \\
\hline & WPP & WPU & WPP & WPU \\
\hline Lower & 9.00 & 7.34 & 6.90 & 5.91 \\
\hline Upper & 6.16 & 4.98 & 4.54 & 4.63 \\
\hline
\end{tabular}

*Perfusion indexes are nameless numbers. In the applied method an increase in index value indicates worsening of perfusion

Several years' study and analysis in our institute allowed us to define for diagnostic purposes a range of normal values for WPP and WPU at rest in young patients and in patients more than 50 years of age (Table I). The results of these values were compared to those obtained in previous studies [5].

We also examined the pulse, calculated anklebrachial indexes $(\mathrm{ABI})$, measured the blood flow maximal speed $\left(V_{\max }\right)$, and indicated the pulse index $(\mathrm{PI})$ and resistance index (RI) in the selected arteries in the typical locations.

\section{Statistical analysis}

All the calculations were performed with the STATISTICA PL 8 software (StatSoft, Poland). The average value and standard deviation were given for the measurable variables. The hypothesis of normal distribution was verified with the ShapiroWilk test. The test power for the radioisotopic indexes was defined, and the correlation between the ankle-brachial index and lower-limb perfusion indexes was calculated as well as correlations between eye fundus fluorescein angiography and perfusion indexes of the lower-limb muscles. The sensitivity and specificity of the utilized method were determined.

\section{Results}

\section{Eye fundus fluorescein angiography results}

Fundus fluorescein angiography allows for qualitative assessment of eye fundus lesions, including in diabetic retinopathy. Repeatable results were obtained for the particular grades of diabetic retinopathy advancement in the conducted study.

Due to the repeatability of the obtained results it was possible to assign significant numerical values (DV) to the grades of diabetic retinopathy advancement.

Assigned numerical values (DV) allowed for statistical analysis of the relationship between the advancement of the diabetic retinopathy and lowerlimb muscle perfusion disturbances.

There were distinguished four characteristics repeating the descriptions of the fundus fluorescein angiography results in group I (NPDR). These are presented in Table II.

There were distinguished three characteristics repeating the descriptions of the fundus fluorescein angiography results in group II (P/PDR). These are presented in Table III.

Mean numerical values (DV) of all the included patients were statistically analysed. The mean numerical value (DV) of each patient was calculated from the mean numerical values (DV) obtained for the left and right eye of each individual patient.

Table II. Group I (NPDR) fundus fluorescein angiography results and DV numerical values

\begin{tabular}{|lc|}
\hline Fundus fluorescein angiography description & (DV) numerical value \\
\hline $\begin{array}{l}\text { Presence of any (i.e. even a small number) of the microaneurysms. } \\
\text { Lack of any other lesion characteristic for diabetic retinopathy }\end{array}$ & 1 \\
\hline $\begin{array}{l}\text { Presence of the higher number of microaneurysms, small number of intraretinal hemorrhages, } \\
\text { any hard exudates, any IRMA'S, any venous changes }\end{array}$ & 2 \\
\hline $\begin{array}{l}\text { Presence of large number of microaneurysms, hard exudates, IRMA'S, venous changes, } \\
\text { intraretinal hemorrhages (less than 20), any cotton wool spots }\end{array}$ & 3 \\
\hline $\begin{array}{l}\text { Presence any of lession } \\
\text { - intraretinal hemorrhages in } 4 \text { retina quadrants } \\
\text { - venous caliber abnormalities such as: venous beading, loops and dilation }\end{array}$ & 4 \\
\hline
\end{tabular}

Table III. Group II (P/PDR) fundus fluorescein angiography results and DV numerical values

\begin{tabular}{|lc|}
\hline Fundus fluorescein angiography description & (DV) numerical value \\
\hline Presence of any NVE of NVD & 1 \\
\hline Simultaneous presence of NVD covering < $1 / 4 D^{*}$ area and NVE covering < 1/2 DD* & 2 \\
\hline Presence of NVD covering $>1 / 4 D^{*}$ area and/or NVE covering $>1 / 2 D^{*}$ & 3 \\
\hline
\end{tabular}

${ }^{\star} D D$ - disc diameter 


\section{Radioisotopic results}

The Shapiro-Wilk test confirmed the normal distribution hypothesis for the perfusion indexes at rest in all groups.

\section{Group I - NPDR (Figure 1)}

In the subgroup of 25 patients treated with oral medication, $7(25 \%)$ had worsening of calf perfusion, and 2 (8\%) had worsening of thigh perfusion. The perfusion index values were in the range $6.67<\mathrm{WPP}<9.85$ and $5.14<\mathrm{WPU}<7.64$. In the insulin subgroup of 18 patients, worsening calf perfusion was observed in 12 (67\%), and in 11 (61\%) thigh perfusion worsened. The perfusion index values were in the range $8.87<$ WPP $<10.56$ and $7.06<\mathrm{WPU}<8.99$.

\section{Group II - P/PDR (Figure 2)}

In the subgroup of 18 patients who were treated with oral medications, 13 (66\%) had worsening perfusion of the calf (WPP) and thigh (WPU). The perfusion index values were in the range 8.65 $<$ WPP < 12.92 and $6.77<$ WPU < 10.97. In the insulin subgroup of 24 patients, worsening calf perfusion was observed in 20 (83\%) and worsening thigh perfusion in 19 (79\%). The perfusion index values were in the range $7.05<\mathrm{WPP}<13.44$ and $6.01<\mathrm{WPU}<11.16$.

The calf perfusion index (WPP) and thigh perfusion index (WPU) in the subgroups underwent the test power assessment.

The perfusion indices of the calf (WPP) and thigh (WPU) in the subgroups underwent the test power assessment. With the zero hypothesis, Mi1 = Mi0, and in relation to the lower-limb perfusion index values in the group of patients more than 50 years of age without the lower-limb perfusion disturbances defined on the basis of clinical examinations, a very high test power was achieved for all cases presented in Table IV.

The small number of patients does not allow for the indication of reliable statistical significance of differences between the perfusion indices in the subgroups treated orally vs. those treated with insulin.

There was a positive correlation between lowerlimb muscle perfusion and fundus diabetic retinopathy results. The strength of this correlation was average in all the NPDR group and high in all the P/PDR group (Table V).

Calculated perfusion indexes allowed us to define sensitivity and specificity of the analysed interrelation between advancement of diabetic retinopathy and lower-limb muscles' perfusion impairment. The results are presented in Table VI.

Characteristic for group I (NPDR) was relatively low sensitivity and very high specificity of the method. Moreover, with increasing advancement of diabetic retinopathy (group II - P/PDR), high sensitivity and also high specificity were observed.

\section{Results of vascular examinations}

Figure 3 presents the distribution of the resistance index $(\mathrm{RI})$, pulse index $(\mathrm{PI})$, and the maximal speed of blood flow (Vmax.) measured in the femoral artery and the ABI. Along with an increased progression of retinopathy, greater dispersion of the values was observed, and the indices increased beyond the normal range.

The power of correlation between the $\mathrm{ABI}$ and the calf perfusion index (WPP) and thigh perfusion

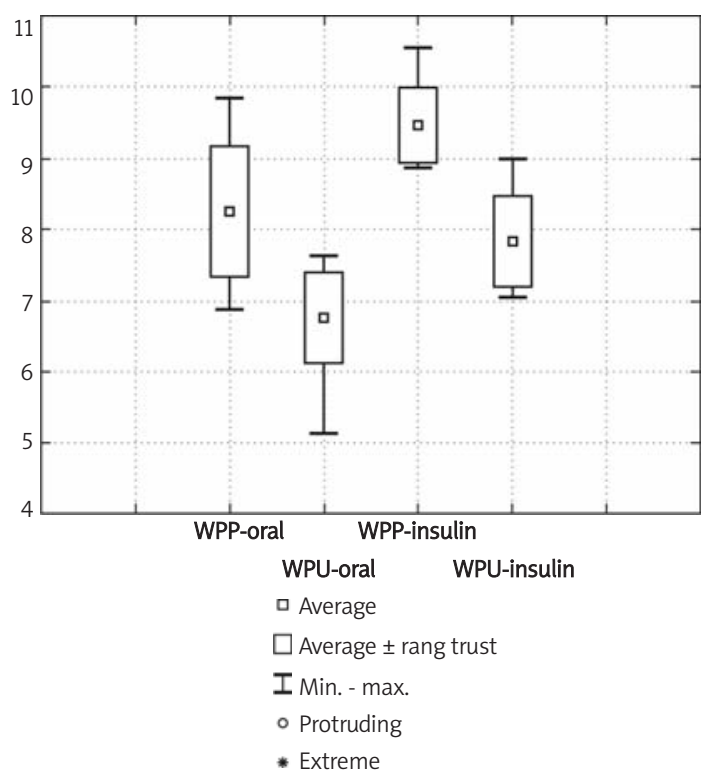

Figure 1. Distribution of results of radioisotopic examination in group 1 - NPDR

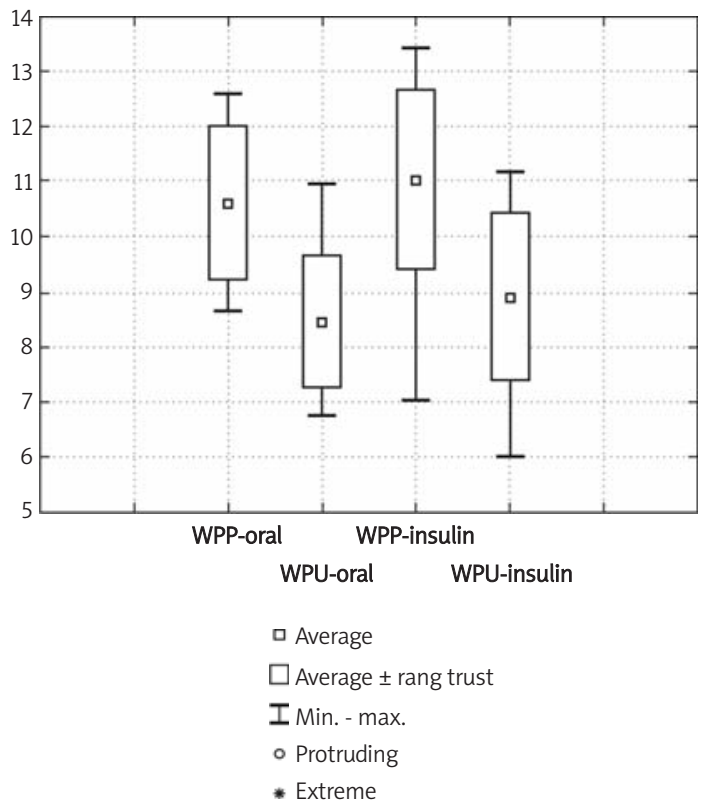

Figure 2. Distribution of results of radioisotopic examination in group $2-P / P D R$ 
Table IV. Assessment of the test power of the analyzed indexes

\begin{tabular}{|lcccccccc|}
\hline & \multicolumn{4}{c}{ Group I - NPDR } & \multicolumn{3}{c|}{ Group II - P/PDR } \\
\cline { 2 - 9 } & \multicolumn{2}{c}{ Oral treatment } & \multicolumn{2}{c}{ Insulin treatment } & \multicolumn{2}{c|}{ Oral treatment } & Insulin treatment \\
\cline { 2 - 9 } & WPP & WPU & WPP & WPU & WPP & WPU & WPP & WPU \\
\hline Population mean (Mi) & 8.2440 & 6.7500 & 9.4570 & 7.8270 & 10.621 & 8.4480 & 11.020 & 8.9040 \\
\hline SD (Sigma) & 0.9237 & 0.6380 & 0.5340 & 0.6320 & 1.4030 & 1.2030 & 1.6300 & 1.5100 \\
\hline number (N) & 25.000 & 25.000 & 18.000 & 18.000 & 24.000 & 24.000 & 24.000 & 24.000 \\
\hline Power & 0.9315 & 0.9933 & 1.0000 & 1.0000 & 1.0000 & 1.0000 & 1.0000 & 1.0000 \\
\hline
\end{tabular}

power; one mean, test $t$, HO: Mi1 = MiO; $p<0,05$

Table V. Relationship between the results of radioisotopic indices of the lower limb muscle perfusion and fundus diabetic retinopathy results

\begin{tabular}{|lllllllll|}
\hline & \multicolumn{4}{c|}{ Perfusion index vs. fluorescein angiography (DV) } \\
\cline { 2 - 9 } & \multicolumn{4}{c}{ Group I - NPDR } & \multicolumn{4}{c|}{ Group II - P/PDR } \\
\cline { 2 - 9 } & \multicolumn{2}{c}{ Oral treatment } & \multicolumn{2}{c|}{ Insulin treatment } & Oral treatment & Insulin treatment \\
\cline { 2 - 9 } & WPP & WPU & WPP & WPU & WPP & WPU & WPP & WPU \\
\hline Correlation (r Pearson) & 0,49 & 0,52 & 0,65 & 0,64 & 0,74 & 0,70 & 0,77 & 0,74 \\
\hline
\end{tabular}

Table VI. Sensitivity and specificity of the method in the particular studied groups

\begin{tabular}{|lcccc|}
\hline & \multicolumn{2}{c|}{ Group I-NPDR } & \multicolumn{2}{c|}{ Group II - P/PDR } \\
\hline & Oral treatment & Insulin treatment & Oral treatment & Insulin treatment \\
\hline Sensitivity [\%] & 47.2 & 64.1 & 75.6 & 82.9 \\
\hline Specificity [\%] & 92.0 & 87.4 & 78.7 & 81.2 \\
\hline
\end{tabular}

index (WPU) is presented in Table VII. It was found that correlation power increased along with the progression of retinopathy. In the simple retinopathy (NPDR) group a lack of correlation was found in the subgroup treated orally. In the insulin subgroup of the NPDR group and in both subgroups of the proliferative and pre-proliferative retinopathy (P/PDR) group, an average correlation power between the indices was observed. The observed correlation was negative, which means that the dependency between the ankle-brachial and perfusion indexes was inversely proportional. A stronger correlation of the $\mathrm{ABI}$ and calf perfusion indexes was observed than between the $A B I$ and thigh perfusion indexes. This may result from the fact that the $A B I$ is calculated on the basis of the pressure measured in the dorsal artery of the calf. The absolute values of the muscle perfusion indexes increased, while the value of the anklebrachial index decreased, which confirmed worsening circulation.

\section{Discussion}

Diseases of peripheral vessels are a common complication of type 2 diabetes. In patients with diabetes, the risk of vascular disease is increased by advanced age, smoking, hypertension, lipid metabolism disturbances, diabetes duration, and the presence of peripheral neuropathy [6-11]. The real frequency of lower-limb disturbances of muscle perfusion is difficult to assess because of a lack of, or a small number of, symptoms in a large group of patients. Hiatt et al. reported that in a group of patients with vascular disease, more than $50 \%$ did not claim any symptoms, about $1 / 3$ had intermittent claudication, and only the remaining ones had more severe forms of the disease [12]. However, even in asymptomatic patients, perfusion disturbances of the lower limbs indicate systemic vascular disease [10, 12-17]. Microangiopathic and macroangiopathic disturbances may be prognostic for the course of muscle vascular diseases in patients with diabetes, and numerous observations suggest the presence of a common pathological mechanism of disturbance. Cosson et al. demonstrated the presence of diabetic retinopathy in patients with type 2 diabetes [15]. In our study we analysed the mutual correlation of microangiopathic and macroangiopathic disturbances in patients with type 2 diabetes and the relation of the frequency of co-existence of sight complications to disturbances of lower-limb peripheral perfusion. Applied non-invasive diagnostic methods that allow for early recognition of lower-limb ischemia clinically and prognostically benefit patients with type 2 

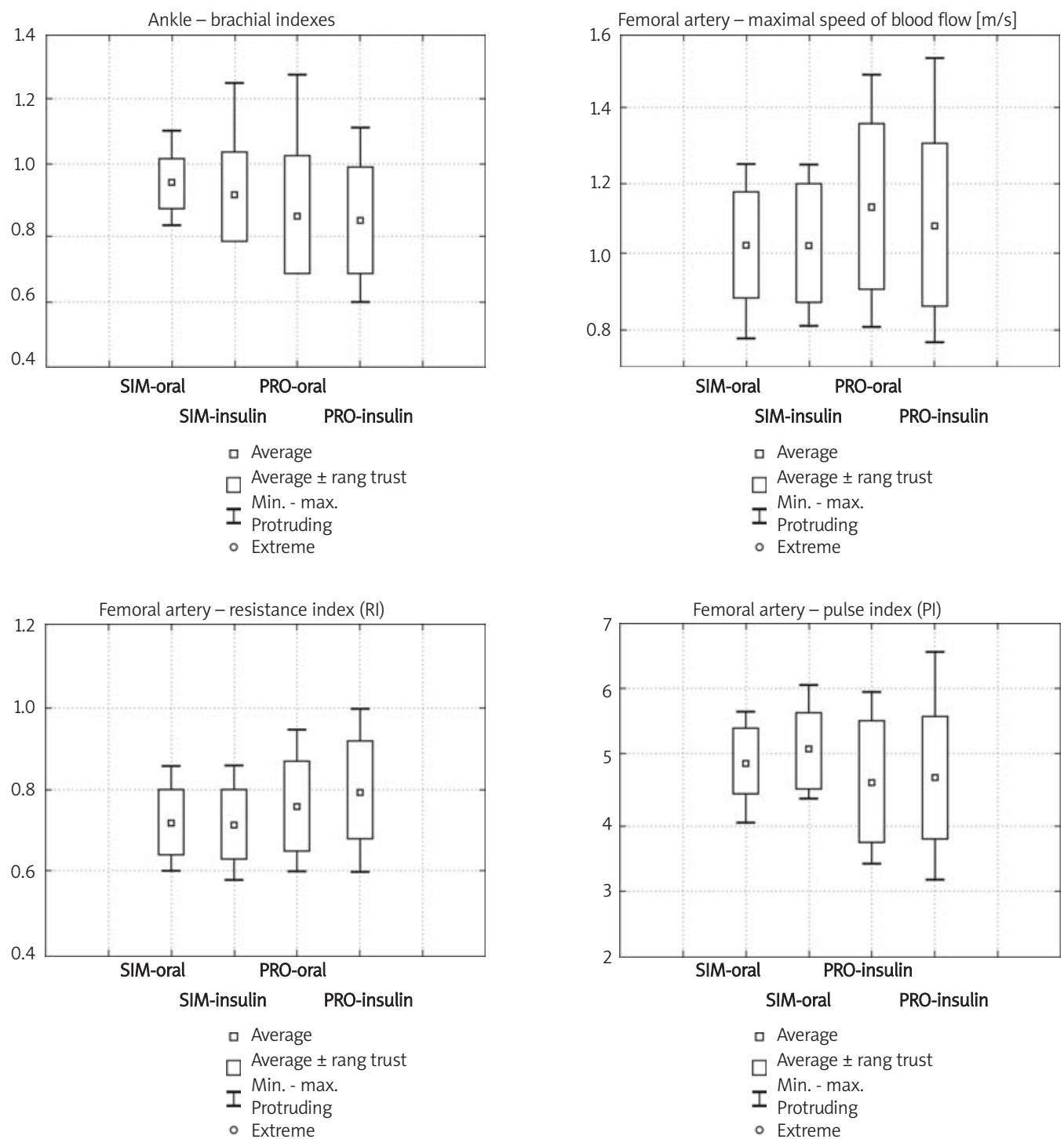

Figure 3. Distribution of basic results of vascular examination in the subgroups

Table VII. Value of the correlation between perfusion and ankle-brachial indexes

\begin{tabular}{|c|c|c|c|c|c|c|c|c|}
\hline & \multicolumn{8}{|c|}{ Perfusion index vs. ankle-brachial index } \\
\hline & \multicolumn{4}{|c|}{ Group I - NPDR } & \multicolumn{4}{|c|}{ Group II - P/PDR } \\
\hline & \multicolumn{2}{|c|}{ Oral treatment } & \multicolumn{2}{|c|}{ Insulin treatment } & \multicolumn{2}{|c|}{ Oral treatment } & \multicolumn{2}{|c|}{ Insulin treatment } \\
\hline & WPP & WPU & WPP & WPU & WPP & WPU & WPP & WPU \\
\hline Correlation ( $r$ Pearson) & $-0,17$ & $-0,02$ & $-0,53$ & $-0,41$ & $-0,63$ & $-0,60$ & $-0,68$ & $-0,49$ \\
\hline
\end{tabular}

diabetes in whom such vascular complications are advanced and often recognized too late. The current literature does not clearly answer the question whether progression of diabetic retinopathy is an important indicator of increased risk of vascular disease $[16,18,19]$. Lower-limb ischemia may develop in $20 \%$ to $50 \%$ of patients with type 2 diabetes, and ophthalmological examinations seem useful in the detection of early changes in peripheral vessels in such patients $[19,20]$. Our study aimed to answer the question whether ophthalmological examination can determine which patients with type 2 diabetes should be evaluated for peripheral perfusion disturbances, even with a lack of clinical symptoms, and to what extent the progression of eyeground changes correlates with the development of changes in peripheral perfusion. A finding of such dependencies would define which patients with type 2 diabetes should have a lowerlimb examination. 
The indexes of the standard vascular examinations significantly exceeded normal values. The ankle-brachial index decreased according to the advancement of the diabetic retinopathy. The results of the analysed vascular examinations suggested impairment of the lower-limb muscle perfusion but gave no possibilities for precise quantification of the defects. The application of perfusion scintigraphy in the study allowed us to supplement former vascular diagnostic methods and to evaluate precise disease advancement.

Relatively low sensitivity (47.2\%) and very high specificity (92\%) of the research method in group I (NPDR) - with oral treatment - showed that not all the patients in this group had lower-limb muscle perfusion disturbances although all the patients had diabetic retinopathy.

In group II (P/PDR) sensitivity and specificity of the method were high and increased along with the advancement of the diabetic retinopathy, which can be of prognostic value and an indication for lowerlimb perfusion assessment.

The patients in our study in whom there were characteristic changes, especially those with retinopathy treated with insulin, should undergo ophthalmological assessment for muscle perfusion disturbances. Our study appears to logically explain such a strategy, which can be useful in the detection of muscle perfusion disturbances in the course of diabetes.

Conclusions are as follows:

1. Deterioration of lower-limb muscle perfusion follows advancement of diabetic retinopathy.

2. There are dubious indications for lower-limb muscle perfusion assessment in group I (NPDR) according to the low sensitivity (50\%) and high specificity of the utilized methods.

3. There are clear indications and high prognostic value of the eye fundus examination according to the lower-limb muscle perfusion disturbances in group II (P/PDR).

4. Diabetic retinopathy advancement degree is a significant indication for lower-limb muscle perfusion assessment despite the lack of subjective complaints characteristic for lowerlimb muscle ischaemia.

5. There are reasonable arguments to implement our suggested strategy to uncover lower-limb perfusion disturbances before the appearance of significant clinical symptoms characteristic for micro- and macroangiopathy.

\section{References}

1. Fong DS, Aiello L, Gardner TW, et al. Diabetic retinopathy. Diabetes Care 2003; 26: 99-102.

2. Harrison DK, Voss C, Vollmar HS, Koutsiaris AG, Newton DJ. Response of muscle oxygen saturation to exercise, measured with near infrared spectrophotometry in patients with peripheral vascular disease. Adv Exp Med Biol 1998; 454: 45-52.

3. Dziuk M, Cholewa M. Scyntygrafia perfuzyjna w ocenie żywotności mięśnia sercowego ze szczególnym uwzględnieniem redystrybucji talu-201. Lek Wojsk 2000; 76: 5-9.

4. Dąbrowski J, Górski A, Mikosiński J, Joss A, Brykalski D. Zastosowanie scyntygrafii perfuzyjnej z użyciem 99mTc MIBI do oceny wyników leczenia przewlekłego niedokrwienia tętniczego kończyn dolnych. Acta Angiol 1996; 4: 249-54.

5. Tryniszewski W, Gadzicki M, Maziarz Z. Determination of normal values range for lower limbs muscles perfusion indexes at rest and after exercise in patients after 50 years of age, with the possibility of assessment of age influence on these values in case of their clinical application.

6. Schmidt MI, Duncan BB, Vigo A, et al. Detection of Undiagnosed Diabetes and Other Hyperglycemia States. Diabetes Care 2003; 26: 1338-43.

7. Tuomilehto J, Lindstrom J, Eriksson JG, et al. Prevention of type 2 diabetes mellitus by changes in lifestyle among subjects with impaired glucose tolerance. N Engl J Med 2001; 344: 1343-50.

8. Adler Al, Stevens RJ, Neil A, Stratton IM, Boulton AJM, Holman RR. UKPDS 59: Hyperglycemia and other potentially modifiable risk factor for peripheral vascular disease in type 2 diabetes. Diabetes Care 2002; 25: 894-9.

9. American Diabetes Association/Consensus Statement: Peripheral arterial disease in people with diabetes. Diabetes Care 2003; 26: 3333-41.

10. Araszkiewicz A, Zozulińska-Ziółkiewicz D, Trepińska M, Wierusz-Wysocka B. Why does intensive insulin therapy implemented at the onset of type 1 diabetes not decrease prevalence of diabetic microangiopathy? Arch Med Sci 2008; 4: 167-73.

11. Kłysik AB, Naduk-Kik J, Hrabec Z, Goś R, Hrabec E. Intraocular matrix metalloproteinase 2 and 9 in patients with diabetes mellitus with and without diabetic retinopathy. Arch Med Sci 2010; 6: 375-81.

12. Hiatt WR. Medical treatment of peripheral arterial disease and claudication. N Engl J Med 2001; 334: 1608-21.

13. Uyarel H, Cam N, Ergelen M, et al. Contrast-induced nephropathy in patients undergoing primary angioplasty for acute myocardial infarction: incidence, a simple risk score, and prognosis. Arch Med Sci 2009; 5: 550-8.

14. Rysz J, Banach M, Stolarek RA, et al. Serum matrix metalloproteinases MMP-2 and MMP-9 and metalloproteinase tissue inhibitors TIMP-1 and TIMP-2 in diabetic nephropathy. J Nephrol 2007; 20: 444-52.

15. Cosson E, Paycha F, Teiller P, et al. Lower-limb vascularisation in diabetic patients. Diabetes Care 2001; 24: 870-4.

16. Leibson CL, Ransom JE, Olson W, Zimmerman BR, O'Fallon WM, Palumbo PJ. Peripheral arterial disease, diabetes, and mortality. Diabetes Care 2004; 27: 2843-9.

17. Norman PE, Davis WA, Bruce DG, Davis TME. Peripheral arterial disease and risk of cardiac death in type 2 diabetes. Diabetes Care 2006; 29: 575-80.

18. National Diabetes Fact Sheet: general information and national estimates on diabetes in the United States, 2005. U.S. Department of Health and Human Services, Centres for Disease Control and Prevention.

19. Tryniszewski W, Gadzicki M, Rysz J, Banach M, Maziarz Z. The behavior of bone mineral density and bone metabolism index in young and menopausal women with the consideration of body mass index. Med Sci Monit 2010; 16: CR342-7. 
20. Klein R, Klein BE, Moss SE, Davis MD, DeMets DL. The Wisconsin epidemiologic study of diabetic retinopathy. II. Prevalence and risk of diabetic retinopathy when age at diagnosis is less than 30 years. Arch. Ophthalmol 1984; 102: 520-6.

21. Klein R, Klein BE, Moss SE, Cruickshanks KJ. The Wisconsin epidemiologic study of diabetic retinopathy. XVII. The 14-year incidence and progression of diabetic retinopathy and associated risk factors in type 1 diabetes. Ophthalmology 1998; 105: 1801-15. 